\title{
Niclosamide attenuates inflammatory cytokines via the autophagy pathway leading to improved outcomes in renal ischemia/reperfusion injury
}

\author{
LIN-XIA ZHANG ${ }^{1}$, HUI-JUAN ZHAO ${ }^{2}$, DONG-LI SUN ${ }^{2}$, SHAN-LIN GAO ${ }^{2}$, \\ HONG-MEI ZHANG ${ }^{2}$ and XIN-GUO DING ${ }^{2}$
}

Departments of ${ }^{1}$ Geriatrics and ${ }^{2}$ Nephrology, Tangshan Gongren Hospital, Tangshan, Hebei 063000, P.R. China

Received December 10, 2015; Accepted January 5, 2017

DOI: $10.3892 / \mathrm{mmr} .2017 .6768$

\begin{abstract}
Renal ischemia/reperfusion (I/R) injury is a debilitating condition that leads to loss renal function and damage to kidney tissue in the majority of patients with acute kidney disease. Previous studies have indicated that autophagy serves a protective function in renal I/R injury. In the present study, the effect of the anthelmintic niclosamide in the regulation of inflammatory responses in kidney I/R was investigated. A total of 40 Sprague-Dawley rats were randomly divided into the following 5 groups $(n=8$ in each group): Sham group; renal I/R injury; renal I/R injury plus 3-methyladenine (3-MA) treatment $(15 \mathrm{mg} / \mathrm{kg})$; renal $\mathrm{I} / \mathrm{R}$ injury plus niclosamide $(25 \mathrm{mg} / \mathrm{kg})$; and renal $\mathrm{I} / \mathrm{R}$ injury plus rapamycin (10 mg/kg). The expression levels of autophagy-associated proteins in kidney samples obtained from rats with I/R injury were examined using reverse transcription-quantitative polymerase chain reaction and western blotting techniques. In addition, histopathological alterations, the expression of cytokines and renal function were evaluated. Treatment with niclosamide was associated with induction of autophagy and an overall improvement in renal function. There was an increased expression of autophagosome-associated proteins, suggesting a strong correlation between autophagy and improvement of renal function. The increased levels of anti-inflammatory cytokines and decreased levels of pro-inflammatory cytokines provided additional evidence that niclosamide may be effective for the treatment of renal I/R injury. Clinical studies are required to further validate the results of the present study.
\end{abstract}

Correspondence to: Dr Hui-Juan Zhao, Department of Nephrology, Tangshan Gongren Hospital, 27 Wenhua Road, Lubei, Tangshan, Hebei 063000, P.R. China

E-mail: zhaohuijuanzhj@hotmail.com

Key words: niclosamide, autophagy, acute kidney injury, inflammation, cytokines, renal ischemia, renal reperfusion injury

\section{Introduction}

Renal ischemia/reperfusion (I/R) injury is the primary etiopathological phenomenon that leads to acute renal failure or multiple organ failure in patients with renal transplant or renal resection $(1,2)$. Acute kidney injury $(\mathrm{AKI})$ is an additional consequence of renal I/R injury, and is responsible for the high number of patients with long-term kidney dysfunction that require intensive medical care (3). Renal I/R injury is characterised by a pathological phenomenon associated with restriction in the blood supply to the kidney, which leads to limited arterial blood flow and an imbalance in the supply of metabolites thatleads to tissue hypoxia (4-10). A reperfusion procedure is employed to restore blood flow and reduce further tissue injury and reduce the inflammatory response (11). Recent advancements in treatment strategies have failed to address the issue of morbidity and mortality in patients undergoing renal transplant (12). Notably, higher mortality rates have been observed in males than females (13). The pathogenesis of renal I/R injury involves a complex combination of inflammation, oxidative stress, autophagy, apoptosis and immunological pathways (14-16). A cascade of inflammatory cytokines, such astumour necrosis factor- $\alpha$ (TNF- $\alpha$ ) and interleukin (IL)-6, have been demonstrated to initiate the recruitment of leukocytes and additional antigen presenting cells that induce a potent immune response $(17,18)$. In addition, renal vascular endothelial cells have been demonstrated to serve a dominant role in recruiting immune cells (19). However, the precise series of events that initiates an inflammatory cascade and subsequent renal damage remains unknown.

Autophagy is an important mechanism by which eukaryotic cells maintain homeostasis in response to various types of stress $(20,21)$. Autophagy is characterised by the presence of an autophagosome; a double-layer membrane organelle in the cytoplasm that breaks down dysfunctional cells, proteins and cell organelles via fusion of the autophagosome with lysosomes, enzymatic digestion proteins or organelles (22). Niclosamide is an inhibitor of the signal transducer and activator of transcription 3 protein that suppresses phosphorylationof signal transducer and activator of transcription 3at the Tyr705 site, and is an approved anthelmintic drug $(23,24)$. A previous study demonstrated that niclosamide is a potent 
enhancer of autophagy and induces mitochondrial fission (25). In addition, nuclear factor- $\kappa \mathrm{B}$, reactive oxygen species, Notch, Wnt/ $\beta$-catenin and mechanistic target of rapamycin complex 1 are additional factors targeted by niclosamide, which suggests that the drug may be useful for the treatment of a number of disorders (26). Previous studies have demonstrated that inductionof autophagy in the proximal (and associated) region of renal tubules during renal I/R injury and acute kidney diseasemay have beneficial effects on the renal tissue (27-29). Therefore, it is necessary to investigate the autophagic properties of niclosamide in a model of renal I/R injury. The present study was performed to investigate the in vivo effects of niclosamide in a rat model of renal I/R injury, and to examine the possible mechanisms of action.

\section{Materials and methods}

Animals. All animal procedures were performed according to the guidelines of the Care and Use of Laboratory Animals (30), and were approved by the Animal Ethical Care and Use Committee of the Tangshan Gongren Hospital (Tangshan, China). A total of 40 male Sprague-Dawley (SD) rats (8 week old, weight 220-250 g) were purchased from the Chinese Academy of Medical Sciences (Beijing, China). Rats were housed in a 12:12 h light:dark cycle (lights on 6am-6pm) with controlled temperature $\left(21 \pm 2^{\circ} \mathrm{C}\right)$ and humidity $(60 \pm 10 \%)$. Sterile water and food were provided to the rats ad libitum.

Experimental design. The SD rats were randomly divided into the following 5 treatment groups ( $\mathrm{n}=8$ in each group): Sham group; renal I/R injury; renal I/R injury plus 3-methyladenine (3-MA) treatment $(15 \mathrm{mg} / \mathrm{kg})$; I/R injury plus niclosamide $(25 \mathrm{mg} / \mathrm{kg})$; and $\mathrm{I} / \mathrm{R}$ injury plus rapamycin (10 mg/kg). Niclosamide, 3-MA and rapamycin were sourced from Sigma-Aldrich (Merck KGaA, Darmstadt, Germany). Following drug treatment for $24 \mathrm{~h}$, biopsies of the kidney tissue were taken to examine the level of autophagosome-associated marker proteins, including microtubule-associated protein 1A/1B light chain 3B (LC3-II), beclin-1, Rab7 and lysosome-associated membrane protein 2 (LAMP2) by western blotting analysis. For biochemical analysis, blood was obtained from the inferior vena cava, and kidney function parameters were measured at $24 \mathrm{~h}$ following the I/R injury induction. For measurement of cytokine levels, kidney samples were obtained following sacrifice.

Induction of $I / R$ injury in rats. The rats were anaesthetised using $10 \%$ chloral hydrate (Sigma-Aldrich; Merck KGaA), at a dose of $400 \mathrm{mg} / \mathrm{kg}$. For I/R injuryinduction, the flank incision method was performed (31). Briefly, the rats were first injected with $30 \mathrm{mg} / \mathrm{kg}$ of sodium pentobarbital (Sigma-Aldrich; Merck $\mathrm{KGaA}$ ), followed by a flank incision to remove the right kidney. Using a small vascular clip, the left renal artery and vein were clamped for $30 \mathrm{~min}$ prior to removal, in order to replicate the reperfusion procedure. Using a sterile suture, the abdomen was closed. Throughout the experiments, the rats were maintained at $32^{\circ} \mathrm{C}$ and hydrated with normal saline. A blood and kidney biopsy was conducted $24 \mathrm{~h}$ aftercompletion of the reperfusion procedure. In the sham group, the identical surgical procedures were performed, but without renal artery and vein clamping. Kidney injury score was determined following the previous report (32).

Histological analysis. Kidney tissues were extracted from rats in all treatment groups ( $24 \mathrm{~h}$ after treatment) following intraperitoneal injection of chloral hydrate $(300 \mathrm{mg} / \mathrm{kg})$. The kidney tissues were stained with Masson's trichrome to analyze alterations in the tissue morphology $(30,33)$. Three independent pathologistsanalysed three different tissue sections from rats in each treatment group. Samples were examined under aZeiss Axio Imager A2 m microscope (Carl Zeiss AG, Oberkochen, Germany). The evaluating pathologist was blinded to the study groups.

Assessment of renal function. Following treatment of rats for $24 \mathrm{~h}$, blood samples were obtained from rats in all experimental groups. Serum creatinine and blood urea nitrogen (BUN) levels were evaluated using the Samsung LABGEOPT10 clinical chemistry analyzer (Samsung, Seoul, South Korea) according to the manufacturer's protocol.

Western blot analysis of LC3-II, beclin-1, Rab7 and LAMP2 expression. Proteins from the renal issue were extracted using M-PER Mammalian Protein Extraction Reagent (Thermo Fisher Scientific, Inc., Waltham, MA, USA) and quantified using a Protein Assay kit (Bio-Rad Laboratories, Inc., Hercules, CA, USA) according to the manufacturer's protocol. Proteins $(10 \mu \mathrm{g})$ were separated by $12 \%$ SDS-PAGE and subsequently transferred to polyvinylidene fluoride membranes. Blots were blocked in 5\% skimmed milk for $2 \mathrm{~h}$ at room temperature and then incubated withthe following primary antibodies: Beclin-1 (1:1,000; no. 3738; Cell Signaling Technology, Inc., Danvers, MA, USA,), LC3-I/II (1:1,000; no. ABC929; EMD Millipore, Billerica, MA, USA), RAB7 (1:1,000; ab137029; Abcam, Cambridge, UK), LAMP-2 (1:500; ab203224; Abcam), and beta-actin $\left(1: 1,000 ;\right.$ ab8227; Abcam) overnight at $4^{\circ} \mathrm{C}$. The membranes were washed in PBS 4-5 times and incubated withhorseradish peroxidase-conjugated goat anti-rabbit IgG secondary antibody (1:2,000; no. ab6721; Abcam) for $2 \mathrm{~h}$ at room temperature. The Amersham ECL Western Blotting Detection kit (GE Healthcare Life Sciences, Chalfont, UK) was used todetect protein expression.

Measurement of TNF- $\alpha$, high mobility group box 1 (HMGB1), IL-6 and IL-10 cytokine levels. Using a tissue homogenizer (Thomas Scientific, Swedesboro, NJ, USA), a tissue lysate was prepared and cytokine levels were estimated using commercially available ELISA kits from R\&D Systems, Inc.(Minneapolis, MN, USA) for rat TNF- $\alpha$ (no. SRTA00), IL-6 (no. PR6000B), IL-10 (no. DY522) or the Cloud-Clone Corp. (Houston, TX, USA) for rat HMGB1 (no. SEA399Ra). Experiments were performed and measured according to the manufacturer's protocol.

Immunofluorescence detection of beclin-1-labelled autophagosomes in renal tissues. Kidney samples were obtained from the following five treatment groups: Control group (sham I/R injury); I/R group; the 3-MA treatment group; the niclosamide treatment group; and the rapamycin treatment group. Tissue samples were processed for immunofluorescence staining 
A
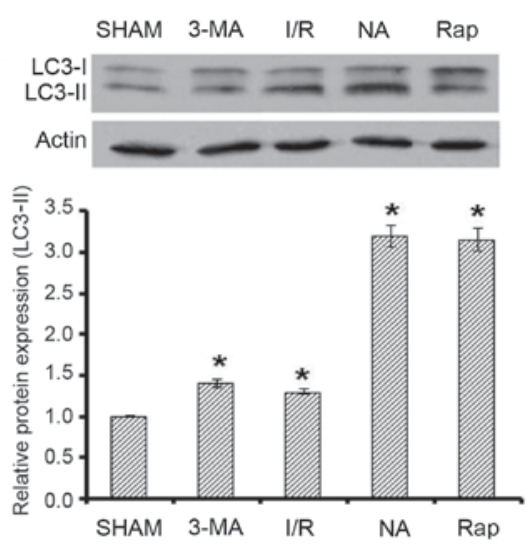

C
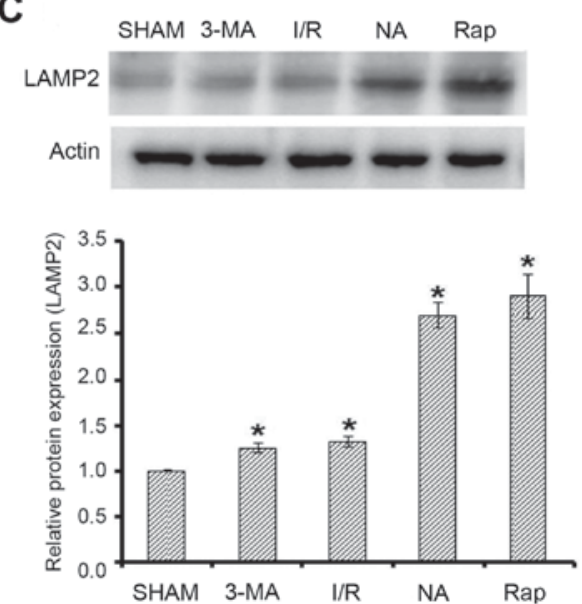

B

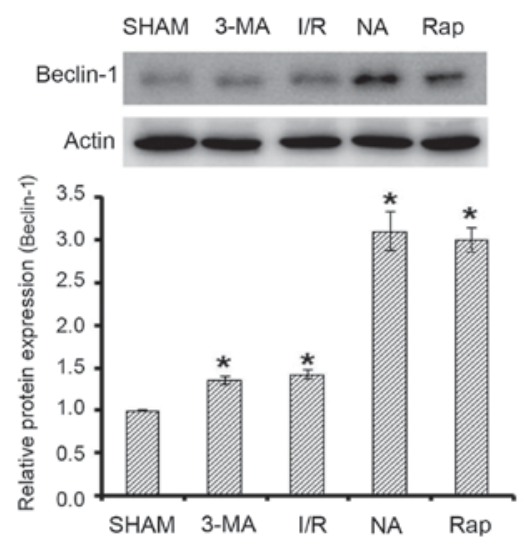

D
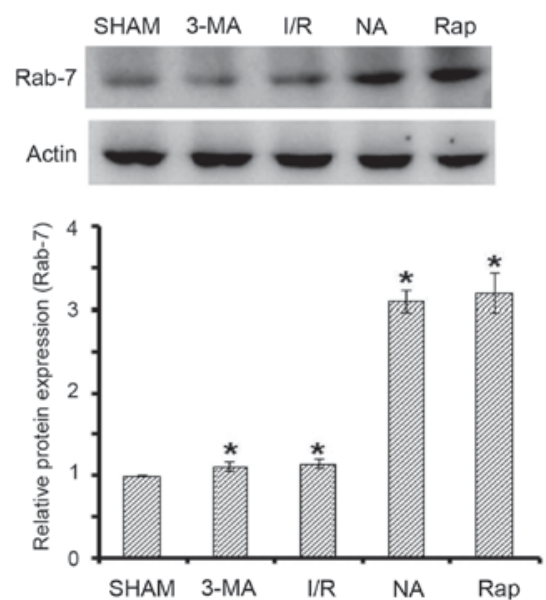

Figure 1. Protein expression levels of (A) LC3-II, (B) beclin-1, (C) LAMP-2 and (D) Rab-7 autophagy-associated markers using western blotting inrenal tissues $(\mathrm{n}=8 /$ group) from rats with ischemia/perfusion injury following treatment with niclosamide $(25 \mathrm{mg} / \mathrm{kg}), 3-\mathrm{MA}(15 \mathrm{mg} / \mathrm{kg}) \mathrm{or} \mathrm{rapamycin}(10 \mathrm{mg} / \mathrm{kg})$. Values are presented as the mean \pm standard deviation. ${ }^{*} \mathrm{P}<0.05$ vs. sham. LC3-II, microtubule-associated protein 1A/1B light chain 3B; LAMP-2, lysosome-associated membrane protein 2; 3-MA, 3-methyladenine; I/R, ischemia/perfusion injury.

for beclin-1 expression using the standard procedure (34). Briefly, kidney tissueswere fixed in $4 \%$ paraformaldehyde overnight and embedded in paraffin. From the paraffin blocks, $5-\mu \mathrm{m}$ sections were cut using a steel microtome. The paraffin sections were then deparaffinised and incubated overnight with a polyclonal anti-beclin-1 antibody (1:200; ab217179; Abcam) overnight at $4^{\circ} \mathrm{C}$. Sections weresubsequently labeled with anti-rabbit Alexa Fluor-488-labeled secondary antibody (no. A32723; Thermo Fisher Scientific, Inc.) at room temperature for $2 \mathrm{~h}$. Photomicrographs were obtained using the Zeiss Axio Imager A2 $\mathrm{m}$ microscope with a florescence filter (magnification, x40; Carl Zeiss AG).

Ultrastructural studies. Kidney tissue samples (size, $1 \mathrm{~mm}^{3}$ ), consisting of a section of renal cortex and outer medulla, were obtained from the following treatment groups: Control group (sham I/R injury); I/R group; the 3-MA treatment group; the niclosamide treatment group; and the rapamycin treatment group. They were subsequently fixed in $4 \%$ paraformaldehyde and $1 \%$ glutaraldehyde in a $0.1 \mathrm{M}$ phosphate buffer $(\mathrm{pH} 7.3)$ for $12 \mathrm{~h}$ at $4^{\circ} \mathrm{C}$, before they were washed in sodium cacodylate buffer. The tissues were post-fixed in $1 \%$ aqueousosmium tetroxide $\left(\mathrm{OsO}_{4}\right)$ solution for $2 \mathrm{~h}$ at $4^{\circ} \mathrm{C}$. Tissues were then dehydrated using a graded ethanol series, before they were embedded in standard Spurr resin (Sigma-Aldrich; Merck KGaA). Finally, 60-90-nm thick sections were stained using uranyl acetate and lead citrate, and were examined under a Hitachi H-7500 Transmission Electron Microscope equipped with a Gatan 780 dual-view CCD camera (Hitachi, Ltd., Tokyo, Japan). Samples were visualised under x1,000 and $\mathrm{x} 10,000$ magnifications. The autophagic vacuoles/100 $\mu \mathrm{m}$ of cytoplasm were evaluated using AxioVision 4 software (v 4.8; Zeiss GmbH, Jena, Germany).

Statistical analysis. Statistical analysis was performed using the SPSS software (version 15.0; SPSS Inc., Chicago, IL, USA). To compare the variation of data among groups, one-way analysis of variance was performed, followed by Fisher's least significant difference test for multiple comparisons. $\mathrm{P}<0.05$ was considered to indicate a statistically significant difference.

\section{Results}

Expression of autophagy-associated protein in the renal tissues of rats with renal I/R injury. To evaluate kidney function following I/R injury and to establish a possible association with enhanced autophagy, westudied the expression of autophagy-associated proteins, beclin-1 and LC3-II. Rapamycin was used as positive 
A

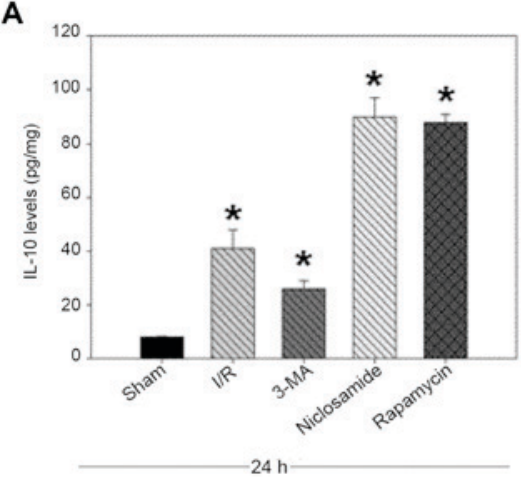

C

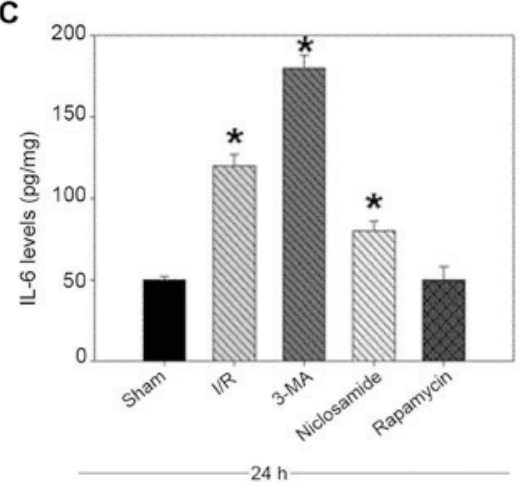

B

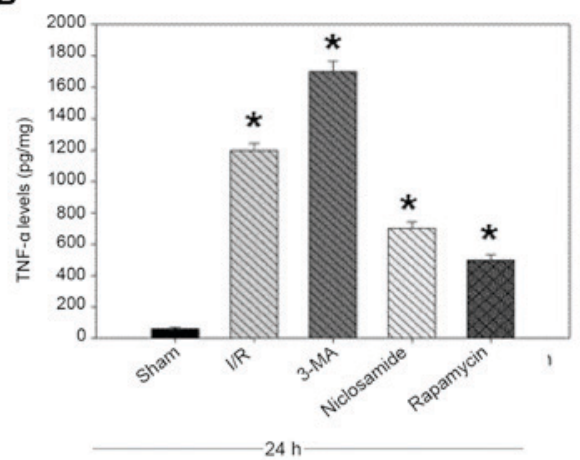

D

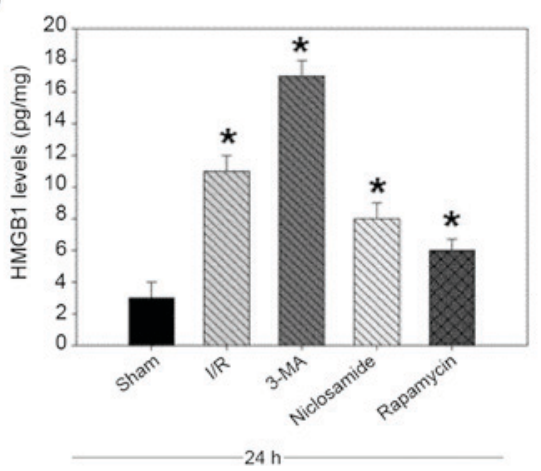

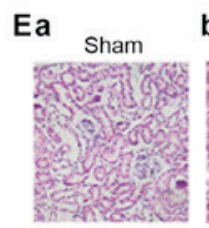
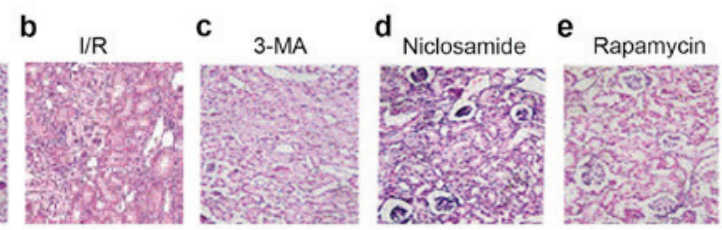

Figure 2. Levels of (A) IL-10, (B) TNF- $\alpha$, (C) IL-6 and (D) HMGB1 in the renal tissues of rats, following renal I/R injury and treatment with niclosamide $(25 \mathrm{mg} / \mathrm{kg}), 3-\mathrm{MA}(15 \mathrm{mg} / \mathrm{kg})$ or rapamycin $(10 \mathrm{mg} / \mathrm{kg})$. (E) Alterations in the histological profile of kidney tissues, following treatment with niclosamide, 3 -MA or rapamycin. Values are presented as the mean \pm standard deviation ( $\mathrm{n}=8 /$ group); ${ }^{*} \mathrm{P}<0.05$ vs. sham. IL, interleukin; TNF- $\alpha$, tumour necrosis factor- $\alpha$; HMGB1, high mobility group box 1; I/R injury, ischemia/perfusion injury; 3-MA, 3-methyladenine.

control, as it is a known activator of autophagy, whereas 3-MA was used as inhibitor of autophagy. An increase in the protein expression levels of beclin-1, LC3-II, LAMP2 and Rab7 in the kidney tissuesfrom rats with renal I/R injury was observed. Treatment with niclosamide resulted in an increase in the expression of beclin-1, LC3-II and lysosome-associated proteins LAMP2 and Rab7, when compared with the sham group ( $\mathrm{P}<0.05$; Fig. 1). After $24 \mathrm{~h}$ of treatment with niclosamide, the increase in the expression of all autophagy-associated markers was comparable with rapamycin treatment (Fig. 1). As expected, a significant reduction in the expression of beclin-1 and LC3-II was observed following treatment with 3-MA compared with the niclosamide- or rapamycin-treated groups. In addition, treatment of rats with I/R injury with 3-MA demonstrated a reduced expression of lysosome-associated proteins LAMP2 and Rab7 compared with the niclosamide- or rapamycin-treated groups, which correlated with the results of subsequent histopathological and electron microscope studies.

Effect of niclosamide on histological alterations and cytokine expression in rats with renal I/R injury. In order to determine the association between renal function with inflammation-mediated autophagy, the levels of pro-inflammatory cytokines TNF- $\alpha$, IL-6, HMGB1 and the anti-inflammatory cytokine IL-10 were measured. Treatment with niclosamide and rapamycin was associated with a reduction in the levels of pro-inflammatory cytokines and an increase in the levels of anti-inflammatory cytokines $(\mathrm{P}<0.05$, Fig. 2A-D). As expected, an increase in the levels of pro-inflammatory cytokines and a decrease in the levels of anti-inflammatory cytokines were observed in the renal tissues of rats treated with the autophagy inhibitor 3-MA compared with the sham group $(\mathrm{P}<0.05$, Fig. 2A-D). These results suggest that induction of autophagy may be associated with increased levels of IL-10 and decreased levels of pro-inflammatory cytokines.

Alterations in the histological profile of kidney samples following treatment with niclosamide, 3-MA and rapamycin were subsequently examined. There was a protuberance in epithelial cells, corticullary and medullary regions, loss of nuclei and degeneration of vacuoles in the kidney tissue of the 3-MA, niclosamide and rapamycin-treated animals with I/R injury (Fig. 2E). In contrast, cells of the untreated group appeared to present normal histological profile. The 
A

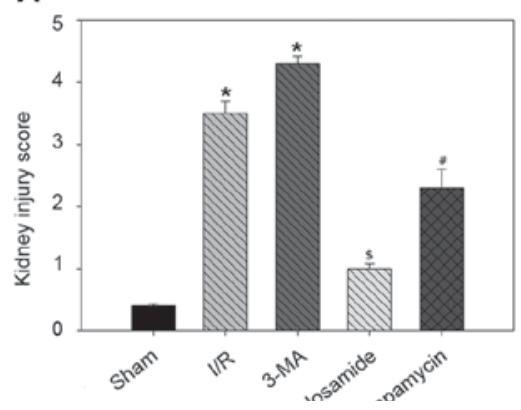

B

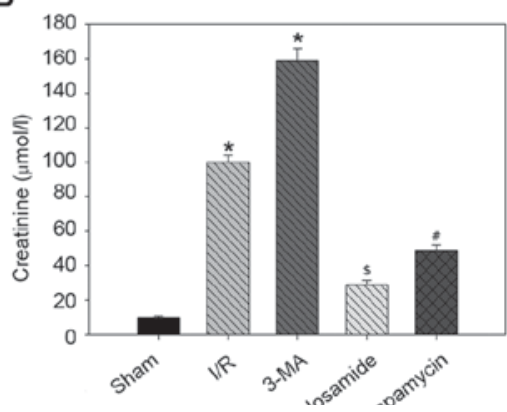

C

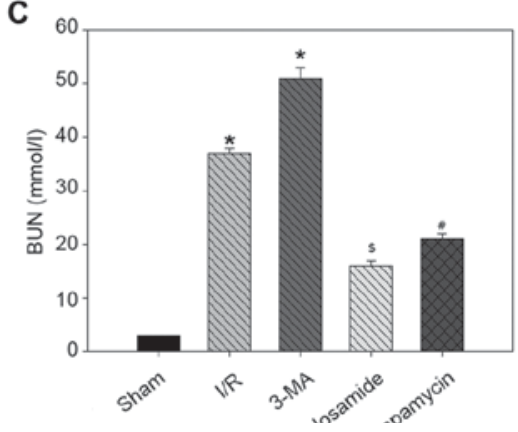

Figure 3. Assessment of (A) renal function and the levels of (B) serum creatinine and (C) BUN in the renal tissues of rats following renal I/R injury and treatment with niclosamide $(25 \mathrm{mg} / \mathrm{kg}), 3-\mathrm{MA}(15 \mathrm{mg} / \mathrm{kg})$ or rapamycin $(10 \mathrm{mg} / \mathrm{kg})$. Values are presented as the mean \pm standard deviation $(\mathrm{n}=8)$. BUN, blood urea nitrogen; I/R injury, ischemia/perfusion injury; 3-MA, 3-methyladenine. ${ }^{*} \mathrm{P}<0.05$ vs. sham, ${ }^{\prime \prime} \mathrm{P}<0.05$ vs. I/R, ${ }^{\mathrm{S}} \mathrm{P}<0.05$ vs. 3-MA.

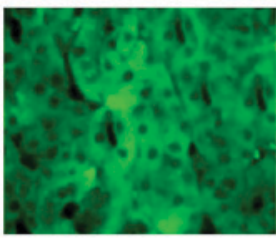

I/R

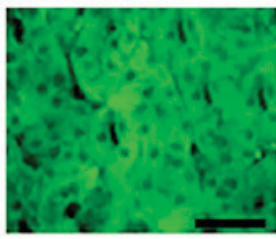

Control

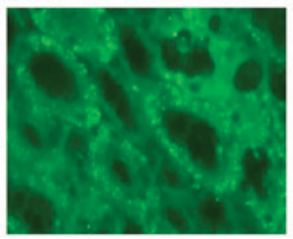

3-MA

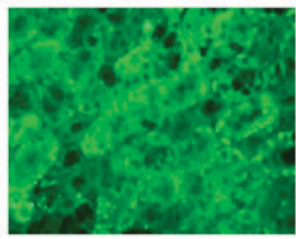

Niclosamide

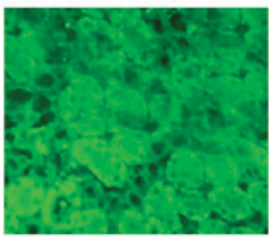

Rapamycin

Figure 4. Immunostaining of beclin-1 expression and induction of autophagy in kidney tissues following renal ischemia/reperfusion injury and treatment with niclosamide $(25 \mathrm{mg} / \mathrm{kg}), 3-\mathrm{MA}(15 \mathrm{mg} / \mathrm{kg})$ or rapamycin $(10 \mathrm{mg} / \mathrm{kg}) .3-\mathrm{MA}, 3$-methyladenine; I/R, ischemia/perfusion injury. Bar, $50 \mu \mathrm{m}$.

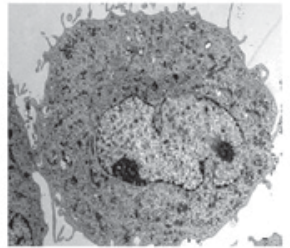

Control

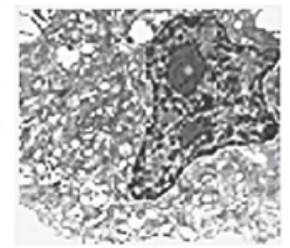

I/R

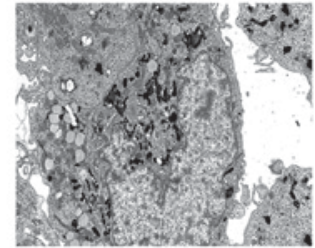

3-MA

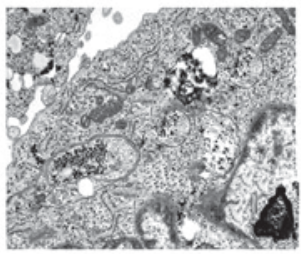

Niclosamide

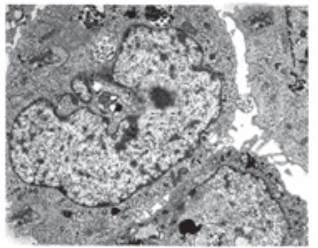

Rapamycin

Figure 5. Ultrastructural analysis of autophagosomes in the renal tissues of rats following renal ischemia/reperfusion injury and treatment with niclosamide (25 mg/kg), 3-MA (15 mg/kg) or rapamycin (10 mg/kg). 3-MA, 3-methyladenine; I/R, ischemia/perfusion injury.

kidney injury score was higher in the treatment groups when compared with the sham group at $24 \mathrm{~h}$ following I/R injury (Fig. 2E and data not shown).

Effect of niclosamide on renal function in rats with $I / R$ injury. The level of kidney function was evaluated by measuring the levels of serum creatinine and BUN. Treatment with niclosamide and rapamycin and was associated with improvedkidney function, as reflected in the decreased kidney injury scorewhen compared with the I/R group (Fig. 3A). In addition, a decrease in the level of creatinine and BUN in niclosamide and rapamycin treatment groups was observed compared with the I/R group $(\mathrm{P}<0.05$; Fig. $3 \mathrm{~B}$ and $\mathrm{C})$. By contrast, treatment with the autophagy inhibitor 3-MA, was associated with an increased kidney injury score (Fig. 3A). When compared with the I/R injury group, creatinine and BUN levels were increased in the 3-MA treatment group (Fig. 3B and C).

In vivo expression of beclin-1 following $I / R$ injury. As shown in Fig. 4, administration of niclosamide $(25 \mathrm{mg} / \mathrm{kg})$ induced a marked increase in the level of beclin-1 when compared with the sham group, and the expression of beclin-1 was comparable to that observed in the rapamycin $(10 \mathrm{mg} / \mathrm{kg})$ treatment group. However, the expression was limited following treatment with the autophagy inhibitor 3-MA $(15 \mathrm{mg} / \mathrm{kg})$. Immunostaining of renal tissues with the beclin-1 antibody indicated clear regions of beclin-1 expression, which is a characteristic indicator of autophagosomes. The expression of beclin-1 around the renal cortical and outer medulla regions was higher in tissues from rats with $\mathrm{I} / \mathrm{R}$ injury when compared with the sham group. Furthermore, beclin-1 expression was higher and more dispersed in the niclosamide group compared withthe I/R group.

Ultrastructural studies. Autophagy levels in renal tissueswereexamined by electron microscopy by examining the number of autophagosomes and additional autophagic vacuoles. No consistent autophagic vacuoles were observed in the shamgroup, however, numerous autophagic vacuoles were observed in the proximal tubular cellsin the treated (I/R) groups (Fig. 5). Under high magnification (x10,000), the autophagic vacuoles possessed a double-membrane structure in the cytoplasm, which is a characteristic of autophagosomes (Fig. 5). In addition, the 
double-membrane structure in the cytoplasm was observed to contain mitochondria and additional cytoplasmic components, includingfragments of the endoplasmic reticulum. Morphometric analysis revealed 1.48 autophagic vacuoles/100 $\mu \mathrm{m}$ cytoplasmin the sham group, 1.2 in the 3-MA group, 2.1 in the I/R group, 5.23 in the niclosamideand 5.9 in the rapamycin treatment groups. Therefore, there was an increased number of autophagosomes in the niclosamide and rapamycin treatment groups.

\section{Discussion}

$\mathrm{I} / \mathrm{R}$ is a major underlying cause of AKI development in native and allograft kidney transplants (34). The incidence of AKIin hospitalized patients has increased, due to the lack of preventive and curative measures (35). The complex pathogenesis of AKI is the result of I/R injury-induced effects on vascular endothelial cells, tubular epithelial cells, and immune cells (36). The effects are characterised by an accumulation of waste products, such as urea and nitrogen, in the cells, alterations in extracellular fluid volume, electrolyte and acid-base balance (35), damage of endothelial cells, necrosis and apoptosis of tubular cells and inflammation (37). In the present study, the effects of niclosamide on renal I/R injury was investigated to determine whether it may serve a protective role by regulating the level of inflammatory cytokines and apoptosis via activation of autophagy in a rat model of renal I/R injury.

Autophagy, a self-destructive response of cells to stress, leads to degradation of endogenous cellular protein aggregates and damaged organelles by lysosomes (38). Previous reports have identified increased levels of autophagy-associated proteins, including LC3-II and beclin-1, in the renal tubules of rats with I/R injury $(26,27)$. LC3 is converted into LC3-I, following proteolytic cleavage at the $\mathrm{C}$-terminal region by autophagy-related protein-4. LC3-I is then transformed by the lipid phosphatidyl ethanolamine to LC3-II. LC3-II is deployed and attaches to the membrane of an autophagosome. LC3-II remains attached to the membrane, until the autophagosome fuses with a lysosome $(39,40)$. Beclin-1 serves a pivotal role in the formation of autophagosome, and has been reported to improve the fusion of the autophagosome and lysosome $(41,42)$. The results of the present study are consistent with earlier reports regarding the activation of autophagy-associated proteins, namely LC3-II and beclin-1, in the renal tubules of rats with I/R injury $(26,27)$. Autophagy is mediated by LC3-II and beclin-1 and a series of regulatory proteins, which serve an important role during the progression of autophagy through different steps; namely the formation of autophagosome, followed by its fusion with lysosome and the final release of degraded products (38). In a rat model of kidney I/R injury, LC3and LAMP2-positive vacuoles were observed to aggregate in cells, thereby indicating fusion between autophagosomes and lysosomes (39). Therefore, LAMP2 may be required for effective fusion $(43,44)$. Additionally, Rab7 has been reported to be required for the fusion of autophagosomes and lysosomes, and for the full development of autophagosomes (45). In the present study, LC3-II, beclin-1, LAMP2 and Rab7 expression levels were increased following induction of renal I/R injury in rats, when compared with the sham group. In the current study, the effects of niclosamide, rapamycin (an activator of autophagy) and 3-MA (an inhibitor of autophagy) were evaluated in a rat model of renal I/R injury. Compared with the sham group, niclosamide and rapamycin treatment increased the expression of LC3-II, beclin-1, LAMP2 and Rab7. In the present study, rats in the I/R injury group demonstrated an increase in kidney injury scores, as well as the levels of creatinine and BUN at $24 \mathrm{~h}$, when compared with the sham group. AKI was associated with the increased expression of autophagy-associated proteins, including LC3-II, beclin-1, LAMP2 and Rab7 at $24 \mathrm{~h}$ following induction of renal I/R injury. Therefore, the results demonstrating then iclosamide-induced increase in the expression of autophagy-associated proteins, decreased kidney injury scores and reduced levels of creatinine and BUN, suggest that it may serve a protective role against renal I/ $\mathrm{R}$ injury in rats, potentially via the induction of autophagy.

Renal I/R injury has been reported to induce an inflammatory response by triggering the innate and adaptive immune systems, followed by infiltration of leukocytes to the site of inflammation and activation tubular epithelial cells $(46,47)$. The infiltration of leukocytes, including neutrophils and macrophages, in the damaged renal tissue promotes the secretion of pro-inflammatory cytokines (48). The infiltrated neutrophils and macrophages reduce blood flow in kidney, resulting dysfunction of the microcirculation $(49,50)$. The levels of pro-inflammatory cytokines, including IL- $1 \beta$, IL- 6 , TNF- $\alpha$ and HMGB1, have been reported to significantly increase following renal I/R injury in rats, which demonstrates that cytokines may be used as an indicator of severity of kidney damage (51). In the present study, rats in the renal I/R injury group demonstrated an increase in the levels of pro-inflammatory cytokines TNF- $\alpha$, IL- 6 and HMGB, and a reduction in the levels of the anti-inflammatory cytokine IL-10 at $24 \mathrm{~h}$ post-renal I/R injury, when compared with the sham group. In the treated renal I/R injury groups, niclosamide and rapamycin decreased the levels of TNF- $\alpha$, HMGB1 and IL- 6 and promoted the release of IL-10 when compared with the untreated I/R group. By contrast, 3-MA treatment was associated with the opposite effect on the expression of these cytokines when compared with the untreated I/R group. Therefore, niclosamide reduces the renal $\mathrm{I} / \mathrm{R}$ injury-induced inflammatory response in AKI.

In conclusion, in a rat model of renal $\mathrm{I} / \mathrm{R}$ injury, niclosamide was observed to induce autophagy and an inflammatory response, as well as decrease kidney injury scores and the levels of creatinine and BUN. This agent may thereforeserve a protective role during AKI by regulating the levels of pro-inflammatory and anti-inflammatory cytokines, potentially via I/R injury-induced autophagy. An exploratory clinical trial involving the incorporation of niclosamide in the treatment protocol will be required to investigate these findings further.

\section{References}

1. Kunzendorf U, Haase M, Rolver L and Haase-Fielitz A: Novel aspects of pharmacological therapies for acute renal failure. Drugs 70: 1099-1114, 2010.

2. Mangano CM, Diamondstone LS, Ramsay JG, Aggarwal A Herskowitz A and Mangano DT: Renal dysfunction after myocardial revascularization: Risk factors, adverse outcomes and hospital resource utilization. The Multicenter Study of Perioperative Ischemia Research Group. Ann Intern Med 128: 194-203, 1998.

3. Malek M and Nematbakhsh M: Renal ischemia/reperfusion injury; from pathophysiology to treatment. J Renal Inj Prev 4: 20-27, 2015. 
4. Aydin Z, van Zonneveld AJ, de Fijter JW and Rabelink TJ: New horizons in prevention and treatment of ischaemic injury to kidney transplants. Nephrol Dial Transplant 22: 342-346, 2007.

5. Chertow GM, Burdick E, Honour M, Bonventre JV and Bates DW: Acute kidney injury, mortality, length of stay, and costs in hospitalized patients. J Am Soc Nephrol 16: 3365-3370, 2005.

6. Kazmers A, Jacobs L and Perkins A: The impact of complications after vascular surgery in veterans affairs medical centers. J Surg Res 67: 62-66, 1997.

7. Levy EM, Viscoli CM and Horwitz RI: The effect of acute renal failure on mortality. A cohort analysis. JAMA 275: 1489-1494, 1996.

8. Zhang J, Li JH, Wang L, Han M, Xiao F, Lan XQ, Li YQ, Xu G and Yao Y: Glucocorticoid receptor agonist dexamethasone attenuates renal ischemia/reperfusion injury by up-regulating eNOS/iNOS. J Huazhong Univ Sci Technolog Med Sci 34: 516-520, 2014

9. Bonventre JV: Daily hemodialysis-will treatment each day improve the outcome in patients with acute renal failure? N Engl J Med 346: 362-364, 2002.

10. Eltzschig HK and Eckle T: Ischemia and reperfusion-from mechanism to translation. Nat Med 17: 1391-1401, 2011.

11. Gabay $\mathrm{C}$ and Kushner I: Acute-phase proteins and other systemic responses to inflammation. N Engl J Med 340: 448-454, 1999.

12. McCaughan JA, Patterson CC, Maxwell AP and Courtney AE: Factors influencing survival after kidney transplant failure. Transplant Res 3: 18, 2014

13. Grams ME and Rabb H: The distant organ effects of acute kidney injury. Kidney Int 81: 942-948, 2012.

14. Hotta O, Yusa N, Ooyama M, Unno K, Furuta T and Taguma Y: Detection of urinary macrophages expressing the CD16 (Fc gamma RIII) molecule: A novel marker of acute inflammatory glomerular injury. Kidney Int 55: 1927-1934, 1999.

15. Akcay A, Nguyen Q and Edelstein CL: Mediators of inflammation in acute kidney injury. Mediators Inflamm 2009: 137072, 2009.

16. Lee DW, Faubel S and Edelstein CL: Cytokines in acute kidney injury (AKI). Clin Nephrol 76: 165-173, 2011.

17. Molitoris BA and Sutton TA: Endothelial injury and dysfunction: Role in the extension phase of acute renal failure. Kidney Int 66 : 496-499, 2004

18. Umehara $\mathrm{H}$, Goda $\mathrm{S}$, Imai $\mathrm{T}$, Nagano $\mathrm{Y}$, Minami $\mathrm{Y}$, Tanaka $\mathrm{Y}$ Okazaki T, Bloom ET and Domae N: Fractalkine, a CX3C-chemokine, functions predominantly as an adhesion molecule in monocytic cell line THP-1. Immunol Cell Biol 79: 298-302, 2001

19. Gottlieb RA and Mentzer RM: Autophagy during cardiac stress: Joys and frustrations of autophagy. Annu Rev Physiol 72: 45-59, 2010.

20. Glick D, Barth S and Macleod KF: Autophagy: Cellular and molecular mechanisms. J Pathol 221: 3-12, 2010.

21. Yorimitsu T and Klionsky DJ: Autophagy: Molecular machinery for self-eating. Cell Death Differ 12 (Suppl 2): S1542-S1552, 2005.

22. Chien CT, Shyue SK and Lai MK: Bcl-xL augmentation potentially reduces ischemia/reperfusion induced proximal and dista tubular apoptosis and autophagy. Transplantation 84: 1183-1190, 2007.

23. Wu HH, Hsiao TY, Chien CT and Lai MK: Ischemic conditioning by short periods of reperfusion attenuates renal ischemia/reperfusion induced apoptosis and autophagy in the rat. J Biomed Sci 16: 19,2009.

24. Suzuki C, Isaka Y, Takabatake Y, Tanaka H, Koike M, Shibata M, Uchiyama Y, Takahara S and Imai E: Participation of autophagy in renal ischemia/reperfusion injury. Biochem Biophys Res Commun 368: 100-106, 2008.

25. Jiang M, Liu K, Luo J and Dong Z: Autophagy is a renoprotective mechanism during in vitro hypoxia and in vivo ischemia-reperfusion injury. Am J Pathol 176: 1181-1192, 2010.

26. Lo S, Yuan SS, Hsu C, Cheng YJ, Chang YF, Hsueh HW, Lee PH and Hsieh YC: Lc3 over-expression improves survival and attenuates lung injury through increasing autophagosomal clearance in septic mice. Ann Surg 257: 352-363, 2013.

27. Hsieh CH, Pai PY, Hsueh HW, Yuan SS and Hsieh YC: Complete induction of autophagy is essential for cardioprotection in sepsis. Ann Surg 253: 1190-1200, 2011.
28. Takahashi W, Watanabe E, Fujimura L, Watanabe-Takano H, Yoshidome H, Swanson PE, Tokuhisa T, Oda S and Hatano M: Kinetics and protective role of autophagy in a mouse cecal ligation and puncture-induced sepsis. Crit Care 17: R160, 2013

29. Wu CT, Sheu ML, Tsai KS, Chiang CK and Liu SH: Salubrinal, an eIF2a dephosphorylation inhibitor, enhances cisplatin-induced oxidative stress and nephrotoxicity in a mouse model. Free Radic Biol Med 51: 671-680, 2011.

30. NRC [National Research Council]: Guide for the Care and Use of Laboratory Animals. 7th edition. Washington DC, National Academy Press, 1996.

31. Wei Q and Dong Z: Mouse model of ischemic acute kidney injury: Technical notes and tricks. Am J Physiol Renal Physiol 303 : F1487-F1494, 2012.

32. Khalid U, Pino-Chavez G, Nesargikar P, Jenkins RH, Bowen T, Fraser DJ and Chavez R: Kidney ischaemia reperfusion injury in the rat: The EGTI scoring system as a valid and reliable tool for histological assessment. J Histol Histopathol 3: 1, 2016.

33. Bellomo R, Kellum JA and Ronco C: Acute kidney injury. Lancet 380: 756-766, 2012

34. Wang X, Du Z,Li L, Shi M and Yu Y: Beclin 1 and p62 expression in non-small cell lung cancer: Relation with malignant behaviors and clinical outcome. Int J Clin Exp Pathol 8: 10644-10652, 2015.

35. Huber TB, Edelstein CL, Hartleben B, Inoki K, Jiang M, Koya D, Kume S, Lieberthal W, Pallet N, Quiroga A, et al: Emerging role of autophagy in kidney function, diseases and aging. Autophagy 8: 1009-1031, 2012

36. Basile DP, Friedrich JL, Spahic J, Knipe N, Mang H, Leonard EC Changizi-Ashtiyani S, Bacallao RL, Molitoris BA and Sutton TA: Impaired endothelial proliferation and mesenchymal transition contribute to vascular rarefaction following acute kidney injury. Am J Physiol Renal Physiol 300: F721-F733, 2011.

37. Linkermann A, Bräsen JH, Himmerkus N, Liu S, Huber TB, Kunzendorf U and Krautwald S: Ripl (receptor-interacting protein kinase 1) mediates necroptosis and contributes to renal ischemia/reperfusion injury. Kidney Int 81: 751-761, 2012.

38. Maiuri MC, Zalckvar E, Kimchi A and Kroemer G: Self-eating and self-killing: Crosstalk between autophagy and apoptosis. Nat Rev Mol Cell Biol 8: 741-752, 2007.

39. Livingston MJ and Dong Z: Autophagy in acute kidney injury. In: Seminars in nephrology. Vol 34. WB Saunders, pp17-26, 2014

40. Hsieh YC, Athar M and Chaudry IH: When apoptosis meets autophagy: Deciding cell fate after trauma and sepsis. Trends Mol Med 15: 129-138, 2009.

41. Mizushima N: Autophagy: Process and function. Genes Dev 21: 2861-2873, 2007.

42. Zhong Y, Wang QJ, Li X, Yan Y, Backer JM, Chait BT, Heintz N and Yue Z: Distinct regulation of autophagic activity by Atg14L and Rubicon associated with Beclin 1-phosphatidylinositol-3-kinase complex. Nat Cell Biol 11: 468-476, 2009.

43. Saftig P and Eskelinen EL: Live longer with LAMP-2. Nat Med 14: 909-910, 2008

44. Zhang YL, Cao YJ, Zhang X, Liu HH, Tong T, Xiao GD, Yang YP and Liu CF: The autophagy-lysosome pathway: A novel mechanism involved in the processing of oxidized LDL in human vascular endothelial cells. Biochem Biophys Res Commun 394: 377-382, 2010

45. Gutierrez MG, Munafó DB, Berón W and Colombo MI: Rab7 is required for the normal progression of the autophagic pathway in mammalian cells. J Cell Sci 117: 2687-2697, 2004

46. Land WG: The role of postischemic reperfusion injury and other nonantigen-dependent inflammatory pathways in transplantation. Transplantation 79: 505-514, 2005.

47. Serteser M, Koken T, Kahraman A, Yilmaz K, Akbulut G and Dilek ON: Changes in hepatic TNF-alpha levels, antioxidant status and oxidation products after renal ischemia/reperfusion injury in mice. J Surg Res 107: 234-240, 2002.

48. Ysebaert DK, De Greef KE, Vercauteren SR, Ghielli M, Verpooten GA, Eyskens EJ and De Broe ME: Identification and kinetics of leukocytes after severe ischaemia/reperfusion renal injury. Nephrol Dial Transplant 15: 1562-1574, 2000.

49. Bolisetty S and Agarwal A: Neutrophils in acute kidney injury: Not neutral any more. Kidney Int 75: 674-676, 2009.

50. Jing XX, Wang ZG, Ran HT, Li L, Wu X, Li XD, Peng XQ, Yang CJ, Li XS and Zhang QX: Evaluation of renal ischemia-reperfusion injury in rabbits using microbubbles targeted to activated neutrophils. Clin Imaging 32: 178-182, 2008.

51. Dessing MC, Pulskens WP, Teske GJ, Butter LM, van der Poll T, Yang H, Tracey KJ, Nawroth PP, Bierhaus A, Florquin S and Leemans JC: RAGE does not contribute to renal injury and damage upon ischemia/reperfusion-induced injury. J Innate Immun 4: 80-85, 2012. 